\title{
SIMULATION OF TURBULENT NATURAL CONVECTION IN A LOCAL ZONE OF LARGE- SCALE AREA UNDER CONDITIONS OF THE RADIANT ENERGY SUPPLY
}

\author{
Alexander $\mathrm{Nee}^{1, *}$, and Liliya Valieva ${ }^{1}$ \\ ${ }^{1}$ National Research Tomsk Polytechnic University, Tomsk, Russia
}

\begin{abstract}
This paper presents the results of mathematical modelling of turbulent natural convection under conditions of the conjugate heat transfer in an open cavity heated by infrared emitter. Two-dimensional problem of heat transfer was formulated in the vorticity - stream function - temperature dimensionless variables and solved by means of the finite difference method on a uniform grid. According to the results of numerical analysis, the fields of differential and integral heat transfer characteristics, illustrating the unsteady nature of the thermophysical process under study, were obtained. The nonlinear dependence of the average Nusselt number versus dimensionless time $(\tau)$ was established. It was shown that the average Nusselt number monotonically increased in a range of $800<\tau<2000$
\end{abstract}

\section{Introduction}

Prediction of thermal regimes of large-scale heat supply objects with radiant heating system is a complex problem. The basic laws of jointly proceeding processes of conduction, convection and radiation under operating conditions of infrared emitters (IE) have not been studied at the level providing energy-efficient use of these systems. At the same time, it is known $[1,2]$ that investigation of heat transfer process in areas with radiant heating sources is advantageously conducted with applying of mathematical modelling methods. However, the development of adequate models of thermophysical processes occurring during IE operating is a challenge. Firstly, the turbulent flow regime should be considered. Secondly, the conjugate heat exchange and radiant energy transfer significantly influence on the microclimate formation in the solution domain. Well-known approaches $[3,4]$ to analyze the thermal regimes of systems with IE performed at a number of assumptions. Thus, the heat transfer model [3] based on the solution of the heat balance equation of the domain does not take into account convective heating of the air and heat-retaining properties of enclosure structures. Investigation [4] conducted under conditions of the laminar flow regime. Moreover, the radiant heating systems in many cases are applied for the local

\footnotetext{
* Corresponding author: nee alexander@mail.ru
} 
heating of specific working areas. Investigation of the heat transfer process in a local domain of large-scale area is of great interest.

The aim of this study is mathematical modelling of turbulent natural convection in a local domain of the large-scale area under conditions of the radiant energy supply.

\section{Problem formulation and solution method}

Local zone (fig. 1) of rectangular cross-section of large-scale area was considered. Solution domain was presented as a cavity filled by air with open boundaries (fig. 2 B1, B2, B3). The radiant flux coming from the IE was uniformly distributed along the air - wall interface (fig. 2 B4). It was assumed that the gas was viscous incompressible and absolutely transparent medium. "Mild" condition [5] was set for the open boundaries. Equalities of temperature and heat fluxes were accepted at the air - wall interface. Investigations were conducted under conditions of the turbulent flow regime.
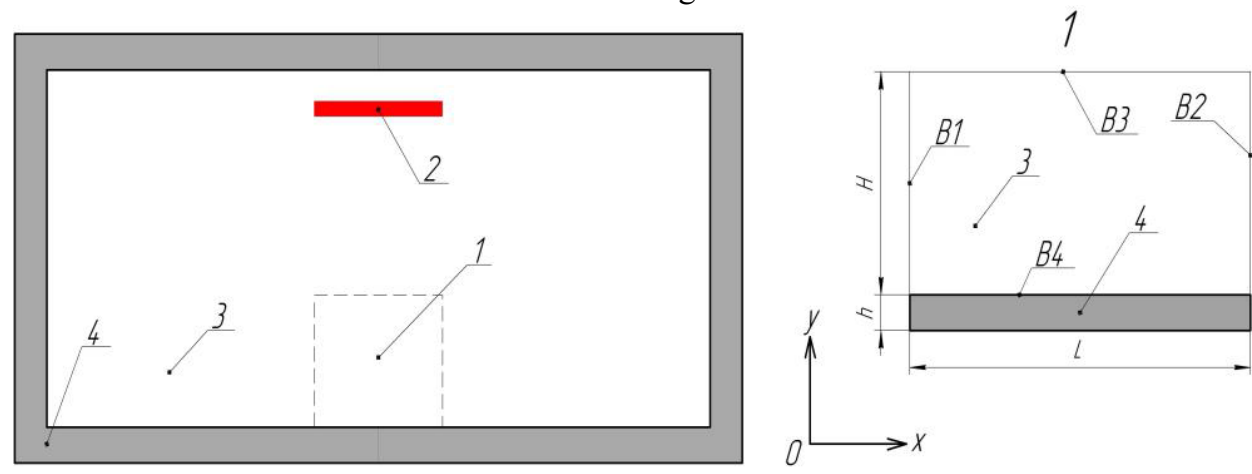

Fig. 1. Solution domain: 1 - the local working zone; 2 - infrared emitter; 3 - the air; 4 - thermally conductive wall; B1, B2, B3 - open boundaries; B4 - the air - wall interface.

Jointly proceeding processes of conduction and turbulent natural convection is described by the Navier - Stokes and energy equations. These equations in the vorticity stream function - temperature dimensionless variables in the Boussinesq approximation are as follows[7]:

$$
\begin{gathered}
\frac{\partial \Omega}{\partial \tau}+U \frac{\partial \Omega}{\partial X}+V \frac{\partial \Omega}{\partial Y}=\frac{\partial^{2}}{\partial X^{2}}\left[\left(\sqrt{\frac{\operatorname{Pr}}{R a}}+v_{t}\right) \Omega\right]+\frac{\partial^{2}}{\partial Y^{2}}\left[\left(\sqrt{\frac{\operatorname{Pr}}{R a}}+v_{t}\right) \Omega\right]+\frac{\partial \Theta_{1}}{\partial X}, \\
\frac{\partial^{2} \Psi}{\partial X^{2}}+\frac{\partial^{2} \Psi}{\partial Y^{2}}=-\Omega, \\
\frac{\partial \Theta_{1}}{\partial \tau}+U \frac{\partial \Theta_{1}}{\partial X}+V \frac{\partial \Theta_{1}}{\partial Y}=\frac{\partial^{2}}{\partial X^{2}}\left[\left(\frac{1}{\sqrt{\operatorname{Ra} \cdot \operatorname{Pr}}}+\frac{v_{t}}{\operatorname{Pr}_{t}}\right) \Theta_{1}\right]+\frac{\partial^{2}}{\partial Y^{2}}\left[\left(\frac{1}{\sqrt{\operatorname{Ra} \cdot \operatorname{Pr}}}+\frac{v_{t}}{\operatorname{Pr}_{t}}\right) \Theta_{1}\right], \\
\frac{\partial \Theta_{2}}{\partial \tau}=F o\left(\frac{\partial^{2} \Theta_{2}}{\partial X^{2}}+\frac{\partial^{2} \Theta_{2}}{\partial Y^{2}}\right) .
\end{gathered}
$$

Subgrid scale eddy viscosity was calculated as in [6]: 


$$
v_{t}=\left(C_{S} \cdot \Delta\right)^{2} \cdot \sqrt{2 \cdot S_{i j} \cdot S_{i j}}, S_{i j}=\frac{1}{2}\left(\frac{\partial U}{\partial Y}+\frac{\partial V}{\partial X}\right) .
$$

The initial conditions for the equations (1) - (4) are as follows:

$$
\begin{gathered}
\Psi(X, Y, 0)=0 ; \Omega(X, Y, 0)=0 ; \\
\Theta_{1}(X, Y, 0)=\Theta_{2}(X, Y, 0)=0 .
\end{gathered}
$$

Boundary conditions for equations (1) - (4) are as follows: at the external boundary of the solid wall:

$$
\frac{\partial \Theta_{2}(X, Y, \tau)}{\partial n}=0
$$

at the air - wall interface:

$$
\Psi=0, \frac{\partial \Psi}{\partial Y}=0,\left\{\begin{array}{l}
\Theta_{2}=\Theta_{1}, \\
\frac{\partial \Theta_{2}}{\partial Y}=\frac{\lambda_{1}}{\lambda_{2}} \cdot \frac{\partial \Theta_{1}}{\partial Y}+K i_{1}
\end{array}\right.
$$

at the open boundaries:

$$
\frac{\partial \Theta}{\partial X}=0, \frac{\partial \Omega}{\partial X}=0, \frac{\partial \Psi}{\partial X}=0,
$$

where $F_{O}-$ Fourier number; $K i-$ Kirpichev number; $\mathrm{Pr}-$ Prandtl number; $\mathrm{Pr}_{t}-$ turbulent Prandtl number; $R a$-Rayleigh number; $U, V$ - dimensionless velocities corresponding $u, v ; X, Y$ - dimensionless coordinates corresponding $x, y ; \tau$-dimensionless time; $\Theta$-dimensionless temperature; $\Psi$ - dimensionless analogue of stream function; $\Omega$ - dimensionless analogue of vorticity; $C_{S}$ - empirical constant (Smagorinsky constant); $\Delta$ - filter width. Indices: 1,2 - elements of the system.

The problem (1) - (4) with the corresponding initial and boundary conditions was solved by means of the finite difference method analogically to $[8,9]$. The implicit four-point pattern [10] was applied to approximate equations (1) - (4). Woods boundary condition [11] was used for the vorticity on a solid no-flip wall. One-dimensional finite-difference analogues of differential equations were solved by means of the sweep method [10].

The solution method and algorithm used were tested on a model problem of turbulent natural convection in a closed square cavity $[12,13]$. Vertical walls were isothermal and horizontal boundaries were assumed adiabatic. Comparison of the fields of temperature and average Nusselt numbers with $[12,13]$ showed their satisfactory agreement.

\section{Results and discussion}

Investigation of differential and integral heat transfer characteristics conducted for the following values of similarity criteria: $R a=10^{8}, \operatorname{Pr}_{t}=1, \operatorname{Pr}=0.71, K i_{1}=42.86$, $K i_{2}=21.43$. Fig. 2 presents the fields of temperature and flow pattern.

Figure 2 shows the fields of differential heat transfer characteristics illustrating the unsteady nature of the thermophysical process under study. It was clearly seen that an 
increase in time led to a rise in temperature at the B4 boundary (fig. 1). When $\tau=300$, two convective plumes (fig. 2 a, c, e) were formed at the air - wall interface. The heated air ascended as the result of natural convection. An increase in time to $\tau=600$ led to merge of convective plumes into the single torch in the section of $\mathrm{X}=0.5$. Air flowed into the cavity through the vertical boundaries (fig. 1 B1, B2) and removed through the horizontal boundary (fig. $1 \mathrm{~B} 3$ ). Convective torch reached the section of $\mathrm{Y}=0.63$. Further increase in the time to $\tau=1200$ led to a rise in the average temperature in the analysis domain. The vertical size of the convective torch increased and reached the top horizontal boundary. The thermophysical process under study entered to the quasi-stationary mode. Further increase in time led to a rise in temperature in the section of $\mathrm{Y}=0.05$ while the air flow pattern and temperature field did not modified. It should be noted that the long-sustained establishment of the quasi-stationary fields of differential characteristics was due to the size of the solution domain corresponding to the turbulent regime of air flow.
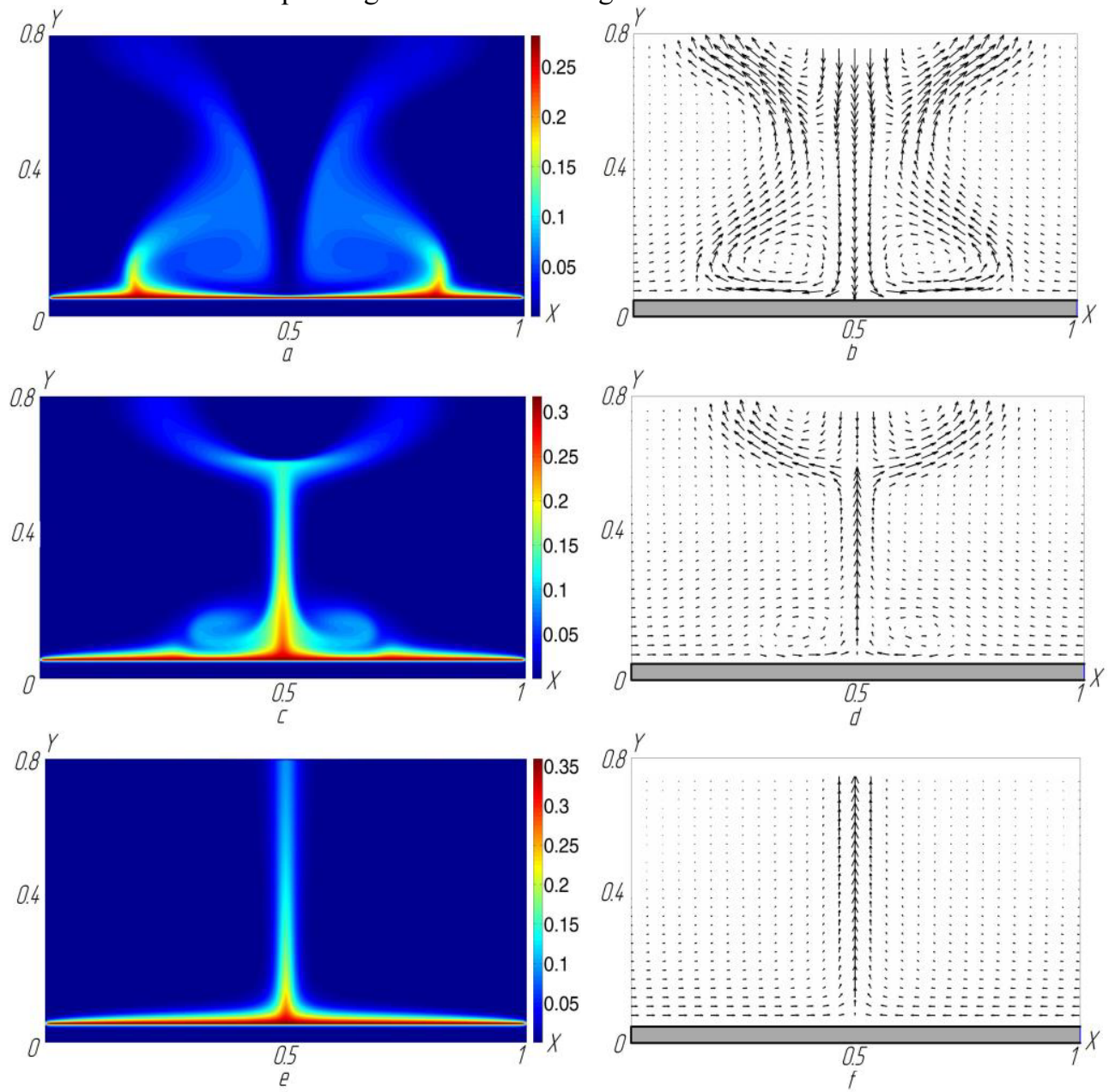

Fig. 2. Fields of temperature (a, c, e) and flow pattern (b, d, f) at $\left.R a=10^{8}: \mathrm{a}, \mathrm{b}\right) \tau=300$;, $\left.\mathrm{d}\right)$ $\tau=600$; e, f) $\tau=1200$.

Fig. 3 shows the temperature distribution in the air cavity in the $\mathrm{Y}=0.5$ section. 


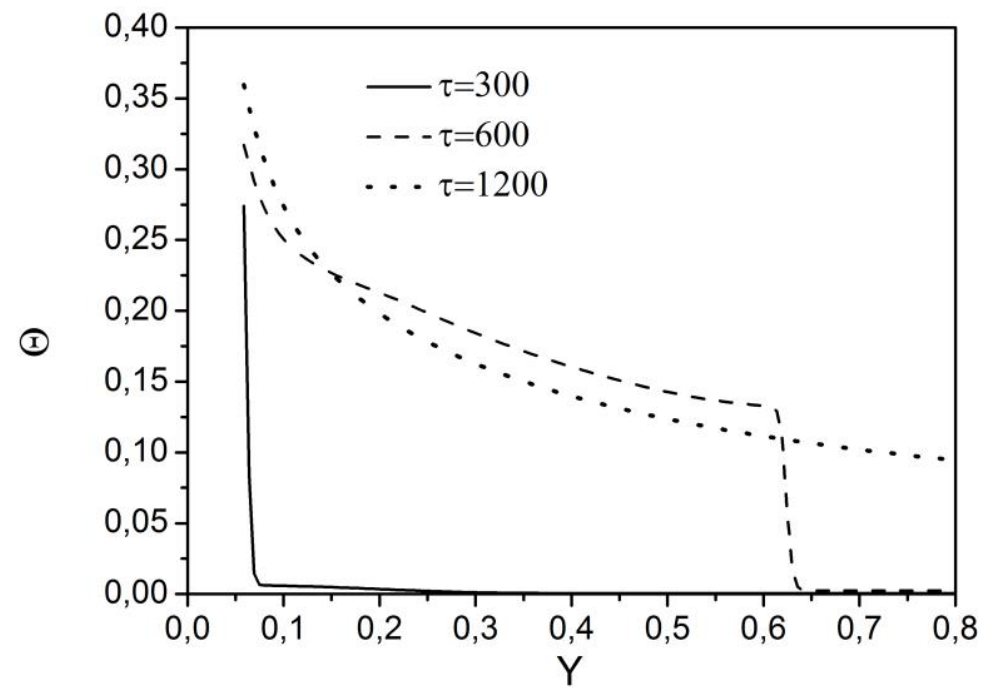

Fig. 3. The temperature distribution in the air cavity in the cross section $X=0.5$.

It was clearly seen that an increase in time led to a rise in temperature at the air - wall interface. The heated air ascended in the direction of the $\mathrm{Y}$-axis. The temperature gradient between the B3 boundary (fig. 1) and air - wall interface was reduced.

Figure 4 presents the dependence of the average Nusselt number at the air - wall interface versus time. The dimensionless heat exchange coefficient was calculated as in $[5$, 9]:

$$
N u_{a v}=\int_{0}^{1}\left|\frac{\partial \Theta}{\partial Y}\right|_{Y=0,05} d X .
$$

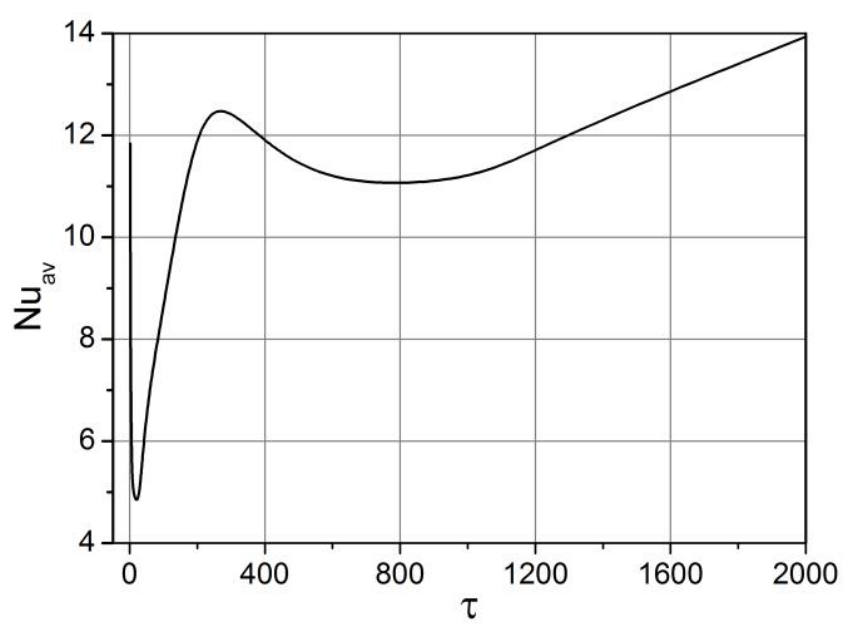

Fig. 4. Dependence of the average Nusselt number versus the dimensionless time. 
Based on the analysis of the presented dependence (fig. 4), it was found that the average integral criterion $\left(N u_{a v}\right)$ was reduced before the time $\tau=30$, which was obviously due to the predominance of conductive heat transfer mechanism over convection. The average Nusselt number increased in a range of $30<\tau<300$, which was connected with the formation of convective plumes at the air - wall interface. A decrease in the average dimensionless heat exchange coefficient in a range of $300<\tau<800$ was due to the formation of the convective torch in the section of $\mathrm{X}=0.5$. Hereafter, the integral criterion $\left(\mathrm{Nu} u_{a v}\right)$ increased monotonically.

\section{Conclusion}

Two- dimensional problem of conjugate turbulent natural convection in the open cavity was formulated and solved. According to the numerical simulation results, it was established that the heat transfer process in a local domain of the large-scale area heated by infrared emitters had significant unsteady nature over a long period of time.

\section{Acknowledgments}

The reported research was supported by Russian Federation President Grant for state support of the Russian Federation leading scientific schools SS-7538.2016.8.

\section{References}

1. G.V. Kuznetsov, V.I. Maksimov, T.A. Nagornova, N.I. Kurilenko, G. Ya. Mamontov, J. of Engin. Ph. and Thermoph. 86, 3 (2013)

2. G.V. Kuznetsov, T.A. Nagornova, A.E. Ni, JEPT. 88, 1 (2015)

3. V. V. Buhmirov, S. A. Krupennikov, Yu. S. Solnyshkova, Her. Ivan. St. Pow. Un., 4 (2010)

4. A. Nee IOP CONF. SER. MATER. SCI. ENG 93 (2015)

5. G.V. Kuznetsov and M.A. Sheremet, Int. J. Heat Mass Transf. 52, 1-2 ( 2009)

6. H. Sajjadi, R. Kefayatib, Therm. Sci. 19, 1 (2015)

7. Sh. Chen, X.-h. Huang, Ch. Yang, G.-f. Liu, C.-j. Ding, B. Han, Appl. Math. Mech. Engl. Ed. 34, 11 (2013)

8. G.V. Kuznetsov, P.A. Strizhak J. Eng. Thermophys-Rus. 17, 3 (2008)

9. G.V. Kuznetsov and M.A. Sheremet, Int J. Heat Mass Transf. 54, 1 - 3 ( 2011)

10. A.A. Samarskii, The Theory of Difference Schemes (Nauka, Moscow, 1977) [in Russian]

11. P. J. Roache, Computational Fluid Dynamics (Hermosa Publishers, Albuquerque, NM, USA, 1976)

12. H.N. Dixit, V. Babu Int J Heat Mass Transf. 49 (2006)

13. N.C. Markatos, K.A. Pericleous, Int J Heat Mass Transf. 27 (1984) 